\title{
In vivo low frequency MR-guided thalamotomy with focused ultrasound: thermal vs mechanical lesioning in pig brain
}

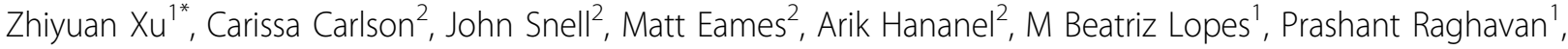 \\ Cheng-Chia Lee ${ }^{1}$, Chun-Po Yen ${ }^{1}$, David Schlesinger ${ }^{1}$, Neal Kassell², Jean-Francois Aubry ${ }^{1,3}$, Jason Sheehan ${ }^{1}$ \\ From Current and Future Applications of Focused Ultrasound 2014. 4th International Symposium \\ Washington, D.C, USA. 12-16 October 2014
}

\section{Background/introduction}

The purpose of this study was to investigate the thresholds for inducing two possible means of tissue destruction with low frequency Magnetic Resonance guided Focused Ultrasound (MRgFUS): either mechanical lesioning in presence of ultrasonic cavitation or pure thermal lesioning (without cavitation).

\section{Methods}

Ten craniectomized pigs where sonicated with an ExAblate4000 Neuro (InSightec, Haifa, Israel) operated with a $220 \mathrm{~Hz}$ array made of 1024 transducers. For each animal, a thermal lesion was aimed on the right thalamus, while a cavitation (mechanical) lesion was aimed on the contralateral side. For thermal lesioning, 40s duration sonications were performed and the acoustical energy ranged between $5600 \mathrm{~J}$ and $12000 \mathrm{~J}$. For mechanical lesioning, 20s duration sonications were performed and similar total acoustic energy was used: energy ranged between $6000 \mathrm{~J}$ and 14000 $J$ (power was increased to compensate for the short sonication time). Signals collected by two passive cavitation detectors (custom made by Insightec) were stored in memory during each sonication and cavitation activity was integrated within the bandwidth of the detectors, from $50 \mathrm{kHz}$ to $182 \mathrm{kHz}$. 2D MR thermometry was performed during treatment. T1-weighted pre- and post-Gadolinium contrast-enhanced, T2-weighted, T2\%-weighted, gradient echo and FLAIR were acquired after treatment. Pigs were euthanized immediately after the last series of MR imaging. Pig brains were harvested and fixed in formalin solution. Histology was performed to identify lesions.

\section{Results and conclusions}

For thermal lesioning, the peak temperature at focus ranged between $49^{\circ} \mathrm{C}$ and $59^{\circ} \mathrm{C}$. All thermal lesions were induced for peak temperature higher than $53^{\circ} \mathrm{C}$. For mechanical lesioning, the peak temperature at focus ranged between $50^{\circ} \mathrm{C}$ and $57^{\circ} \mathrm{C}$. Passive cavitation signals exhibited three main types of signal interpreted as follows: no cavitation, stable cavitation and inertial cavitation. Pure thermal lesions, as assessed by histology, could be generated with low frequency ultrasound. Such lesions showed up on T2 MR post-treatment images as a hypointense core surrounded by a hyperintense ring.

Mechanical lesions where associated with hemorrhages. The size of the hemorrhages measured on gross histology correlated with cavitation activity $(\mathrm{R} 2=0.74)$ and a threshold for cavitation activity of $0.09 \mathrm{~V} . \mathrm{Hz}$ (given the sensitivity of the Insightec cavitation detector or the frequency range) was found to divide the experiments into two separate groups: with and without hemorrhage. This work demonstrates that low frequency ultrasound can induce thermal lesions in the brain of living swines without hemorrhage. This work paves the way towards passive-cavitation-based automatic shutdown of low frequency ultrasound for safe ablation.

\section{Acknowledgements (Funding)}

This work was funded by a grant from the Focused Ultrasound Foundation.

\section{Authors' details}

${ }^{1}$ University of Virginia, Charlottesville, Virginia, United States. ${ }^{2}$ Focued Ultrasound Foundation, Charlottesville, Virginia, United States. ${ }^{3}$ Institut Langevin, Paris, France.

'University of Virginia, Charlottesville, Virginia, United States

Full list of author information is available at the end of the article 
- Convenient online submission

- Thorough peer review

- No space constraints or color figure charges

- Immediate publication on acceptance

- Inclusion in PubMed, CAS, Scopus and Google Scholar

- Research which is freely available for redistribution 